\title{
Clinical impact of a new cardiac magnetic resonance imaging program: a single center US experience
}

\author{
Siddique Abbasi ${ }^{*}$, Jaehoon Chung ${ }^{1}$, Ankit A Desai ${ }^{1}$, Afshin Farzaneh-Far ${ }^{1,2}$ \\ From 15th Annual SCMR Scientific Sessions \\ Orlando, FL, USA. 2-5 February 2012
}

\section{Summary}

While establishing a Cardiac Magnetic Resonance (CMR) program at an academic institution in the United States, we sought to evaluate indications and direct clinical impact of CMR on patient management and decision-making.

\section{Background}

CMR provides a wealth of unique diagnostic and prognostic information in patients with cardiovascular disease. However, little is known about the direct additive effects of information derived from CMR with respect to clinical decision making. Although there is preliminary data suggesting significant impact of CMR on clinical management, this has been derived entirely from European centers where routine use of CMR is significantly more widespread than in the United States. In the current health care environment in the United States, greater use of a new imaging modality like CMR requires evidence for direct additive impact on clinical management.

\section{Methods}

This was a single-center registry from an academic medical center in the United States. The first 200 patients from a newly established CMR program were enrolled. All procedures were performed using standardized SCMR-recommended protocols on $1.5 \mathrm{~T}$ and $3 \mathrm{~T}$ scanners and interpreted by a Level 3 reader. Definitions for "significant clinical impact" of CMR were pre-defined and were collected directly from medical records and/or from patients. Categories of clinical impact included: new diagnosis, medication change, hospital admission/ discharge, as well as performance or avoidance of invasive procedures (angiography, revascularization, device therapy or biopsy).

\section{Results}

The most common indications for referral were cardiomyopathy/myocarditis (28\%), ischemic heart disease/viability testing (19\%), suspected infiltrative disease (11\%), and cardiac mass/thrombus (8\%). Nearly all patients (96\%) had recent prior echocardiograms. In $48 \%$ of patients, CMR had a significant clinical impact. This included an entirely new diagnosis in $25 \%$ of cases and a change in medication management in $12 \%$. CMR results directly led to angiography in $5 \%$ and to performance of percutaneous coronary intervention in $5 \%$. Bypass surgery was performed in $2 \%$ and avoided in $5 \%$, directly as a result of CMR findings.

\section{Conclusions}

This experience demonstrates the ability of a new CMR program to significantly impact clinical decision-making at an academic medical center in the United States. In approximately half of all patients referred, CMR led to a meaningful change in clinical management. This additive impact was seen despite widespread use of echocardiography and other imaging tests. This study lends support to the growing use of CMR in the United States.

\section{Funding}

None. No author disclosures. 


\section{Author details}

'Division of Cardiology, University of Illinois at Chicago, Chicago, IL, USA.

2Division of Cardiology, Duke University, Durham, NC, USA.

Published: 1 February 2012

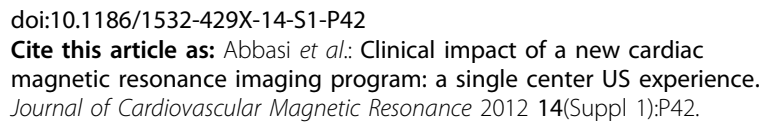

Submit your next manuscript to BioMed Central and take full advantage of:

- Convenient online submission

- Thorough peer review

- No space constraints or color figure charges

- Immediate publication on acceptance

- Inclusion in PubMed, CAS, Scopus and Google Scholar

- Research which is freely available for redistribution 